\title{
Humans' folk physics is not enough to explain variations in their tool-using behavior
}

\author{
FRANCISCO J. SILVA and KATHLEEN M. SILVA \\ University of Redlands, Redlands, California
}

\begin{abstract}
Two experiments examined adult humans' folk physics (i.e., their naturally occurring and spontaneous understanding of the physical world) using variations of trap-table problems used to study chimpanzees' folk physics. When presented with these problems, people unnecessarily avoided retrieving a reward by pulling a rake on the side of a table with a trapping hole-even though it was highly unlikely that the hole would trap the reward. However, when the distance between the reward and the trap was sufficiently large and the distance that the reward had to travel to be retrieved was sufficiently short, people preferred to retrieve a reward by pulling the rake on the side of the table with the trap. These results underscore that behavior during tool-use tasks has many possible causes, only one of which might be a subject's folk physics.
\end{abstract}

Innumerable studies have compared humans' and chimpanzees' genetics, physiology, tool use, social behavior, language, and understanding of various facets of their worlds. One area that has garnered attention recently is chimpanzees' and people's folk physics, defined as a commonsense understanding of the physical world that develops naturally during the course of a subject's life and forms his or her conception of how the world works (Povinelli, 2000). Differences in folk physics (such as the relationship between rigidity and transfer of force) might be evident if a chimpanzee persistently tried to retrieve an out-of-reach banana with a flimsy rope, whereas a person sought the same goal using a rigid rake. The rope leads to failure; the rake leads to success. Researchers who study chimpanzees' folk physics have concluded that these animals, unlike humans, know little if anything about unobservable physical phenomena (e.g., gravity and transfer of force) and thus cannot invoke abstract causal concepts to solve problems (Povinelli, 2000).

One task commonly used to study chimpanzees' and humans' folk physics involves the use of a trap table. In a trap-table problem, two rake tools can be used to drag a reward along either a flat solid surface (successful retrieval) or a flat surface with a trapping hole (unsuccessful retrieval). Pulling the rake on the side with the trap causes the reward to drop into the hole (see Figure 1A). In other variations, one side of the table has a hole in a position that is unlikely to trap a reward (see Figures $1 \mathrm{~B}$ and 1C). For these two configurations, a bias to pull a rake is evidence that the subject did not invoke the abstract concept "gravity" (Povinelli \& Reaux, 2000).

We gratefully acknowledge a discussion with Bill Timberlake that sparked the idea for this study, and the helpful comments of three anonymous reviewers. Please address correspondence to F. J. Silva, Department of Psychology, University of Redlands, P.O. Box 3080, 1200 East Colton Avenue, Redlands, California 92373-0999 (e-mail: francisco_ silva@redlands.edu).
Most chimpanzees do not easily solve trap-table problems, and those that do require considerable trial-and-error learning and/or display unnecessary behavioral biases. In contrast, when people are presented with the schematics shown in Figure 1, they avoid choosing the rake on the side of the table with a hole, even if the hole is unlikely to trap the reward (Silva, Page, \& Silva, 2005). Thus, a bias to avoid an unlikely trap is not necessarily evidence that a subject cannot invoke the appropriate hypothetical physical constructs.

In the experiments reported here, adult humans solved real trap-table problems. The use of adults and simple problems allowed us to manipulate features of a problem and observe the effect on people's choices - all the while knowing that they understood "gravity," "transfer of force," and the like (see, e.g., Silva et al., 2005). In other words, this approach allowed us to determine environment-behavior relationships in a context in which the participants' knowledge of folk physics was transparent. We can then use this information to (1) develop a taxonomy of how different environmental variables affect people's choices on folk physics tasks, (2) suggest future studies with chimpanzees, and (3) provide a context for interpreting chimpanzees' behavior.

\section{EXPERIMENT 1}

The goal of Experiment 1 was to use trap-table problems to evaluate the influence on people's choices of a reward's distance from a potential trap. We examined the distance between the trap and the reward because the participants in Silva et al.'s (2005) study remarked that a hole - even one that was unlikely to trap a reward - had a greater chance of trapping a reward than no hole at all. If this concern is the basis for people's choices in trap-table problems, then it should be possible to mitigate this worry and lessen people's avoidance of the side with the trap by increasing the distance between a reward and a trap. However, if the distance between a reward and a poten- 


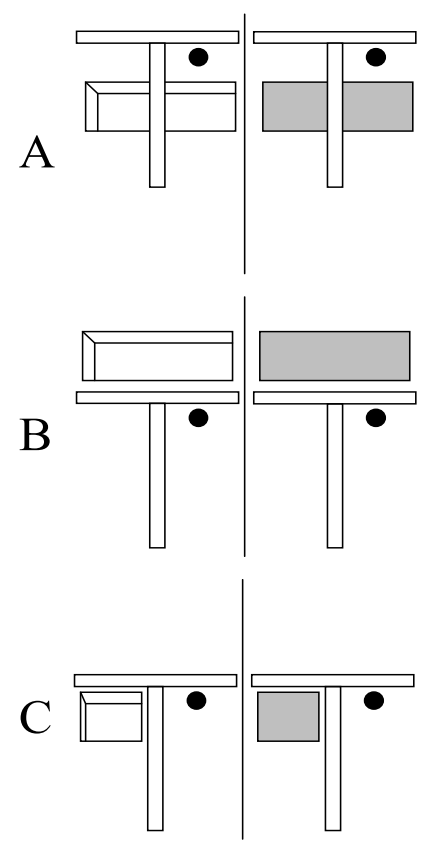

Figure 1. Various configurations of the trap-table apparatus used to study chimpanzees' folk physics (not drawn to scale). (A) A configuration in which pulling the rake on the left causes the reward to fall into the trapping hole, but pulling the rake on the right successfully retrieves the reward. In this configuration and those shown in $B$ and $C$, the gray rectangle or square on the right side is simply a painted surface; it does not interfere with the retrieval of the reward. (B) A configuration in which pulling the rake on either side of the table should successfully retrieve the reward because the trapping hole on the left side is in an ineffective position above the rake and reward. (C) A configuration in which pulling the rake on either side of the table should successfully retrieve the reward because the trapping hole on the left side is in an ineffective position to the left of the reward. From Folk Physics for Apes: The Chimpanzee's Theory of How the World Works (p. 140), by D. J. Povinelli, 2000, Oxford: Oxford University Press. Copyright 2000 by Daniel J. Povinelli. Adapted with permission.

tial trap is large enough that the trap is not perceived as a threat and the distance over which the rake could access the reward is shorter than the distance on the side without a trap, then people should choose to use the rake on the side with the trap. Stated another way, if the reward is sufficiently safe from the potential trap, people should choose to use the rake that minimizes the distance a reward must travel to be retrieved. This variable is an important determinant of people's behavior on another task used to study folk physics, which involves a trap tube. In this task, people (and probably chimpanzees and capuchin monkeys) choose an alternative that minimizes the distance a reward must travel to be retrieved (see Silva et al., 2005, and their analysis of studies by Limongelli, Boysen, \& Visalberghi, 1995; Reaux \& Povinelli, 2000; Visalberghi \& Limongelli, 1994).

\section{Method}

Participants. Thirty-five undergraduate students volunteered for the study and received extra credit for their involvement.
Setting and Apparatus. The trap tables were constructed of 0.48 -cm-thick white foam board supported $5 \mathrm{~cm}$ above the surface of a desk. The seven configurations presented to the participants are shown in Figures 1A and 2A through 2F. The tables measured $70 \times$ $70 \mathrm{~cm}$. For Configuration A in Figure 1 and Configurations A, B, and $\mathrm{C}$ in Figure 2, a $10 \times 30 \mathrm{~cm}$ rectangular hole equidistant from the outside edge and the center of the table and $15 \mathrm{~cm}$ from its top was cut into one side of the table. The other side of the table had a painted (gray) rectangle of the same size and in the same relative position as the hole on the opposite side. For Configurations D, E, and F in Figure 2, a $10 \times 10 \mathrm{~cm}$ square hole whose left edge was $5 \mathrm{~cm}$ from the outside edge of the table and $30 \mathrm{~cm}$ from its top was cut into one side of the table. The other side of the table had a gray square of the same size and at the same relative location. The tables were constructed so that the left and right sides were interchangeable.

The rakes were constructed from a wood dowel $(67 \mathrm{~cm}$ long; $0.63-\mathrm{cm}$ diameter) attached to the center of a $5 \times 35 \mathrm{~cm}$ piece of the same poster board used for the trap tables. M\&Ms served as the rewards; in their farthest and nearest positions, they were placed $38 \mathrm{~cm}$ and $19 \mathrm{~cm}$, respectively, from the bottom edge of the table. The order in which the configurations were presented varied randomly across trials, as did the left-right positions of the traps.

Procedure. All of the participants were tested individually. The experimenter set up a trap table before a participant entered the testing room. After the participant had been asked to enter the room, the experimenter told the participant that the experiment was designed to examine how people use tools to solve problems, and then instructed the participant to choose one of the rakes to drag the reward to the bottom edge of the table. The participants were explicitly instructed to drag the reward slowly and to use whichever hand they preferred in order to pull the rake. Finally, the participants were told
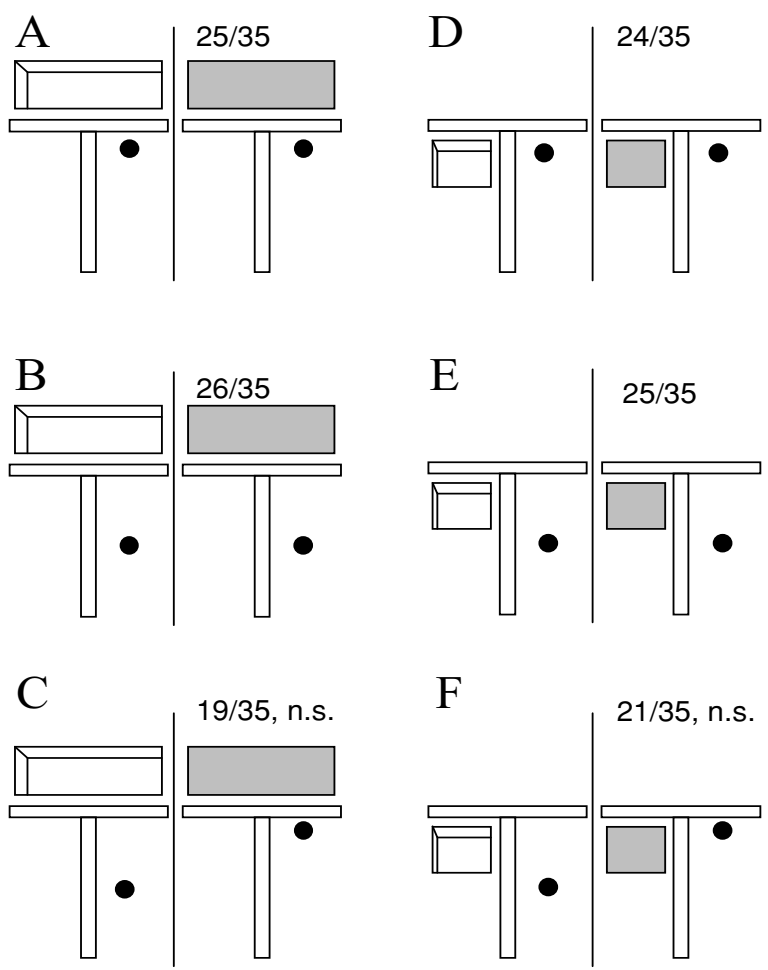

Figure 2. Various configurations of the trap-table apparatus presented to the participants (not drawn to scale). The fraction above the rake shows the number of people who pulled that rake. All outcomes were statistically significant unless denoted with n.s., for nonsignificant. 
that there were multiple solutions for some problems. After a participant made a choice, he or she was asked to step outside of the testing room while the experimenter set up another trap table. After this had been done, the participant was asked to re-enter the room and make another choice. This cycle repeated itself until each of the seven configurations shown in Figures $1 \mathrm{~A}$ and $2 \mathrm{~A}-2 \mathrm{~F}$ had been presented to a participant. Unless otherwise noted, two-tailed binomial tests were used to analyze the results.

\section{Results and Discussion}

All 35 participants obtained the reward from all seven configurations. When the choice was between a rake that could be used to retrieve the reward successfully and a rake that could not (Figure 1A), every person pulled the rake on the side of the table without the trap $(35 / 35, p<.001)$. When the choice was between pulling a rake on the side of the table with or without a trap - and the distance between the rewards on each side of the table and their corresponding traps or painted "traps" was the same (Figures 2A, $2 \mathrm{~B}, 2 \mathrm{D}$, and $2 \mathrm{E}$ ) - most people pulled the rake on the side without the hole $(25 / 35,26 / 35,24 / 35,25 / 35[p s<.05]$ for Configurations A, B, D, and E, respectively). However, when the distance between the reward and the trap was greater than the distance between the reward and a painted "trap" (Figures 2C and 2F), the participants were indifferent about which rake they pulled (19/35 and 21/35 $[p \mathrm{~s}>.31]$ chose the rake on the side without the hole in Configurations $\mathrm{C}$ and $\mathrm{F}$, respectively).

These results show that people, unlike chimpanzees, readily choose the rake that allows a reward to be retrieved without it falling into a trap (Figure 1A). Given the simple tasks used and that everyone successfully solved every trap-table problem, we can be certain that all of the participants understood the abstract causal features embedded in the problems. The results also indicate that, all else being equal, people prefer to retrieve a reward on the side of the table without a hole, even if the hole is unlikely to trap the reward (Figures 2A and 2D). Moreover, and contrary to our prediction, the participants did not lessen their avoidance of the side with the trap when the risk of the hole trapping the reward decreased (Figures 2B and $2 \mathrm{E})$. However, it was possible to weaken the bias to avoid this side. When the distance between the reward and the trap was large enough and the distance over which the rake could access the reward was shorter than the distance on the side without a trap, people were indifferent about which rake they pulled. Had it been only a matter of the distance that the rewards had to travel to be retrieved, the participants should have been indifferent about which rake they pulled in Configurations B and E in Figure 2. That the participants avoided the side of the table with the hole (Configurations B and E) means that they were still concerned about the ability of the hole to trap the reward. Given that the distances between the reward and the trap in Configurations $\mathrm{C}$ and $\mathrm{F}$ of Figure 2 were identical to those in Configurations B and $\mathrm{E}$ of the same figure, any concerns about the potential trap should have been the same. That the bias for avoiding the side with the hole was weakened when the alternative response involved dragging the reward a greater distance suggests that people consider both the distance of the reward from a potential trap and how far the reward must travel to be retrieved. These results are similar to those obtained with trap tubes. With this apparatus, people insert a stick tool into the end of the tube to push out a reward that minimizes the distance the reward travels (Silva et al., 2005).

\section{EXPERIMENT 2}

In Experiment 1, increasing the distance between a reward and a trap caused people to become indifferent about which rake they pulled when the reward on the other (solid) side had to travel a greater distance to be retrieved (Figures 2C and 2F). This effect suggests that, if the distance between a reward and a trap was further increased, the side with the hole should be sufficiently "safe" that people would not avoid this side. However, to produce a bias for the side with the hole, the distance that the reward must travel on this side might have to be much less than the distance that the reward on the solid side must travel. We evaluated this possibility in Experiment 2.

\section{Method}

Participants. Twenty-seven undergraduates volunteered for the study and received extra credit for their involvement.

Setting, Apparatus, and Procedure. The same trap-table apparatus, procedure, and data analyses used in Experiment 1 were used in Experiment 2. The only difference was that the location of the nearest rewards was $7.5 \mathrm{~cm}$ from the bottom edge of the table.

\section{Results and Discussion}

All 27 participants obtained the reward from all seven configurations. When the choice was between a rake that could be used to retrieve the reward successfully and a rake that could not (Figure 1A), every person pulled the rake on the side of the table without the trap $(27 / 27, p<$ $.001)$. When the choice was between pulling a rake on the side of the table with or without a trap - and the distance between the rewards on each side of the table and their corresponding traps or painted "traps" was the same (Figures $3 \mathrm{~A}, 3 \mathrm{~B}, 3 \mathrm{D}$, and $3 \mathrm{E}$ ) — most people pulled the rake on the side without the hole when the reward was near a potential trap $(21 / 27$ and $20 / 27$ [ $p$ s $<.01]$ for Configurations $\mathrm{A}$ and D, respectively). However, when the reward was very far from a trap or painted "trap," people were indifferent about which rake they pulled (15/27 and 16/27 [ $p \mathrm{~s}>.44]$ for Configurations $\mathrm{B}$ and $\mathrm{E}$, respectively).

When the distance between the reward and the trap was large enough and the distance that the reward had to travel on this side was much less than the distance the reward on the solid side had to travel (Figures 3C and 3F), the participants preferred to retrieve the reward on the side with the hole $(25 / 27$ and $23 / 27$ [ $p$ s $<.001]$ for Configurations $\mathrm{C}$ and $\mathrm{F}$, respectively).

These results replicate and add to the results of Experiment 1 . The bias to avoid the side with the trap can be changed to indifference about which rake is pulled by greatly increasing the distance between a reward and a trap or painted "trap" (Figures 3B and 3E). In addition, this indifference can be changed to a bias in favor of the side with 

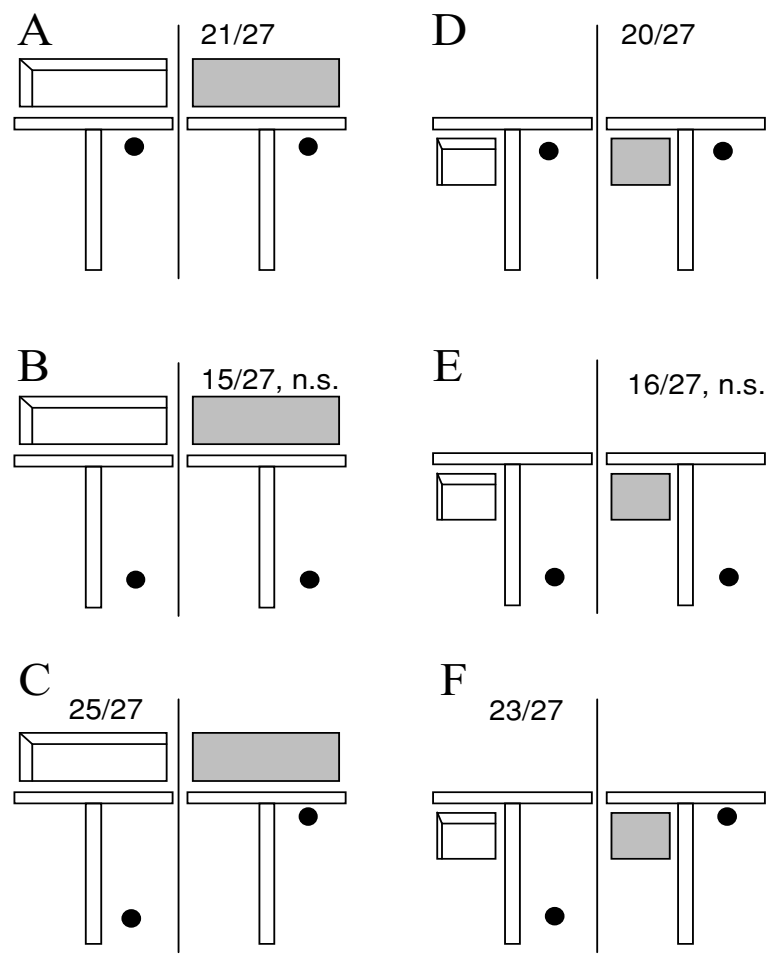

Figure 3. Various configurations of the trap-table apparatus presented to the participants (not drawn to scale). Details are the same as for Figure 2.

the hole by greatly reducing the relative distance that the reward on this side must travel (Figures $3 \mathrm{C}$ and $3 \mathrm{~F}$ ).

\section{GENERAL DISCUSSION}

This study showed that people's choices in the trap-table task were controlled by (1) the presence of a highly effective trap (these were avoided), (2) the presence of a potential but unlikely trap (these were avoided under some circumstances), (3) the distance between a reward and a trap (the greater the distance, the fewer people avoided the side with the trap), and (4) the distance a reward had to travel to be retrieved (if the distance between the reward and the trap was large enough, people preferred to retrieve the reward on this side). Thus, this study identified four variables that control people's responses during trap-table problems and also suggests future experiments that could be conducted with chimpanzees. Also, the results of people's choices on the real trap-table problems presented in Figure 1 replicated the results obtained when schematics of these problems were used (see, e.g., Silva et al., 2005). Thus, whether real or schematic trap tables of similar proportions are used, people seem to focus on the same critical variables. (For a similar result obtained with trap tubes, see Silva et al., 2005.)

That people's behavior changed systematically as a function of the concrete physical characteristics of the task, but not the abstract causal features of the task, shows the importance of obtaining more information about the variables controlling people's and nonhuman animals' behavior in tool-use tasks. Knowledge of humans' folk physics was not enough to predict and explain their tool-using behavior. The participants' behavior seemed to follow a rule (i.e., avoid holes that can trap an object) until another variable (e.g., the distance that the reward had to travel to be retrieved) mediated the influence of the rule. Without more information about the many variables that affect behavior during tool-use tasks, only the most tentative conclusions can be drawn about the nature of a subject's folk physics.

Before more definitive conclusions can be reached about chimpanzees' understanding of physical causality, additional experiments that vary several physical features of the task will be necessary. The present study highlights the interactive effect of a reward's distance from a trap and the distance that a reward must be dragged in trap-table problems. Similar experiments with chimpanzees and monkeys might be informative, as might experiments that vary the initial distance of the reward from the tool, the type of reward used (e.g., a primary reward vs. a token), and whether one or two rakes are available to retrieve the rewards.

It should be possible to extend the methodology used here to the study of the folk physics of chimpanzees (see, e.g., Povinelli, 2000), monkeys (Visalberghi \& Limongelli, 1994), or birds (Chappell \& Kacelnik, 2004). Although the purpose of studying these animals is often to discover what they understand, it should be possible to teach them to understand abstract physical concepts and then conduct experiments to determine how different environment-behavior relationships affect the expression of this understanding (as was done in the present study). Povinelli (2000) tried but did not succeed in training most of his chimpanzees to learn abstract physical concepts; however, his methods were very different from those normally used to teach concepts (e.g., Herrnstein, 1979; see Machado \& Silva, 2003). Concept learning requires many stimulus-response-consequence pairings, in which irrelevant stimulus dimensions are uncorrelated. But Povinelli (2000) avoided using traditional concept learning techniques because, in his view, this would invalidate the goal of studying chimpanzees' folk physics - that is, their naturally occurring understanding of physical causality. Although explicitly trained understanding (i.e., understanding provided by training in an experiment) seems incompatible with naturally occurring understanding, it is questionable whether there is a meaningful distinction between the two (Machado \& Silva, 2003). If there is no meaningful distinction, then folk physics could be redefined as a subject's understanding of physical causality - without any restrictions about how this understanding comes about. With this definition, researchers have the flexibility to train animals whose understanding of folk physics is more transparent and then to study which variables produce different behavioral manifestations of their folk physics. This may ultimately be a better approach than pitting representational views against each other (see, e.g., Povinelli, 2000) or representational views against ecological views (see, e.g., Cummins-Sebree \& Fragaszy, 2005).

To illustrate, imagine that we had compared two representational models with each other, such as a low level of 
understanding with a high level of understanding. A low level of understanding occurs when subjects learn only a "procedural rule, with no accompanying appreciation of one or more of the causal relations involved" (Povinelli \& Giambrone, 2000, p. 116). The subject's behavior is explained by basic learning principles (e.g., stimulus control or consequent learning). In the absence of stimulus generalization training and concept learning, behavior is often context specific. In contrast, a subject who understands the abstract causal relations in a "physics problem" is showing high-level understanding. The knowledge of abstract relations should allow behavior to transfer easily to novel contexts.

Had we followed this approach, our results would have suggested that the participants did not understand the abstract causal principles embedded in the trap table-but only if we based our conclusions on the configurations in Figures $2 \mathrm{~A} / 3 \mathrm{~A}, 2 \mathrm{~B}, 2 \mathrm{D} / 3 \mathrm{D}$, and $2 \mathrm{E}$. With these configurations, people displayed a low level of understanding. However, when presented with the configurations in Figures 3B and 3E, people displayed a high level of understanding. Given that it is inconceivable that the people in the study did not understand the concept "gravity," these seemingly contradictory results underscore the fact that behavior during tool-use tasks has many possible causes, only one of which may be a subject's folk physics.

\section{REFERENCES}

Chappell, J., \& Kacelnik, A. (2004). Selection of tool diameter by New Caledonian crows Corvus moneduloides. Animal Cognition, 7 , 121-127.
Cummins-Sebree, S. E., \& Fragaszy, D. M. (2005). Choosing and using tools: Capuchins (Cebus apella) use a different metric than tamarins (Saguinus oedipus). Journal of Comparative Psychology, 119, 210-219.

Herrnstein, R. J. (1979). Acquisition, generalization, and discrimination reversal of a natural concept. Journal of Experimental Psychology: Animal Behavior Processes, 5, 116-129.

Limongelli, L., Boysen, S. T., \& Visalberghi, E. (1995). Comprehension of cause-effect relations in a tool-using task by chimpanzees (Pan troglodytes). Journal of Comparative Psychology, 109, 18-26.

Machado, A., \& Silva, F. J. (2003). You can lead an ape to a tool, but ... : A review of Povinelli's Folk physics for apes: The chimpanzee's theory of how the world works. Journal of the Experimental Analysis of Behavior, 79, 267-286.

Povinelli, D. J. (2000). Folk physics for apes: The chimpanzee's theory of how the world works. Oxford: Oxford University Press.

Povinelli, D. J., \& Giambrone, S. (2000). Escaping the argument by analogy. In D. J. Povinelli, Folk physics for apes: The chimpanzee's theory of how the world works (pp. 9-72). Oxford: Oxford University Press.

Povinelli, D. J., \& Reaux, J. E. (2000). The trap-table problem. In D. J. Povinelli, Folk physics for apes: The chimpanzee's theory of how the world works (pp. 132-148). Oxford: Oxford University Press.

Reaux, J. E., \& Povinelli, D. J. (2000). The trap-tube problem. In D. J. Povinelli, Folk physics for apes: The chimpanzee's theory of how the world works (pp. 108-131). Oxford: Oxford University Press.

Silva, F. J., Page, D. M., \& Silva, K. M. (2005). Methodologicalconceptual problems in the study of chimpanzees' folk physics: How studies with adult humans can help. Learning \& Behavior, 33, 47-58.

VisAlBerghi, E., \& Limongelli, L. (1994). Lack of comprehension of cause-effect relations in tool-using capuchin monkeys (Cebus apella). Journal of Comparative Psychology, 108, 15-22.

(Manuscript received July 6, 2005; revision accepted for publication September 19, 2005.) 